\title{
Solução de problemas de potencial em meios semi-infinitos bidimensionais através do Método dos Elementos de Contorno
}

\author{
Loeffler, C. F. ${ }^{*}$; Coutinho, F. C. ${ }^{1}$; Lara, L. O. C. ${ }^{1}$ \\ 1 Programa de Pós-Graduação em Engenharia Mecânica, Universidade Federal do Espírito Santo, Vitória, ES, Brasil. \\ *e-mail: loefflercarlos@gmail.com
}

\begin{abstract}
Resumo
O presente trabalho apresenta a formulação e os princípios matemáticos que compõem o modelo do Método dos Elementos de Contorno para a abordagem de problemas bidimensionais com meios infinitos e semi-infinitos, incluindo as particularidades referentes à definição das variáveis pertinentes, às características das soluções fundamentais nestes casos e o comportamento das variáveis primais em pontos infinitamente distantes. Os aspectos relacionados à discretização e às questões numéricas também são abordados, através da solução de dois problemas particulares envolvendo semi-planos.
\end{abstract}

\begin{abstract}
The present work presents the formulation and mathematical principles that comprise the Boundary Element Method model to approach two-dimensional problems with infinite and semi-infinite domains. The particulars regarding the definition of the relevant variables, the characteristics of the fundamental solutions in these cases, and the behavior of primal variables at infinitely distant points are discussed. Aspects related to the discretization and numerical issues are also addressed by solving two particular semi-plane problems.
\end{abstract}

Keywords: Boundary Element Method, Infinite Domains, Semi-Infinite Domains.

\section{Introdução}

Comparativamente aos demais métodos numéricos baseados no conceito de discretização do contínuo, o Método dos Elementos de Contorno (MEC) apresenta grandes vantagens, a mais importante delas prescindir da discretização no domínio do sistema, realizando-a apenas no seu contorno (vide Fig. 1) [1]. A entrada de dados é muito simplificada e de fácil reestruturação, o que o faz adequado aos problemas de fronteira móvel, fratura, contato e concentração de esforços. Devido à sua estrutura matemática, seu desempenho numérico apresenta notável precisão, sobretudo no cálculo de valores no interior do domínio.

Contudo, uma vantagem especial do MEC consiste do tratamento de problemas ditos abertos, ou seja, seu domínio é infinito ou semi-infinito [2]. A discretização de problemas dessa natureza através de técnicas de domínio é um difícil desafio, pois, sem uma estratégia adequada, a abordagem tradicional geraria equações em grande número, tornando o custo de sua solução inviável. Já o MEC elimina matematicamente a discretização de regiões distantes.

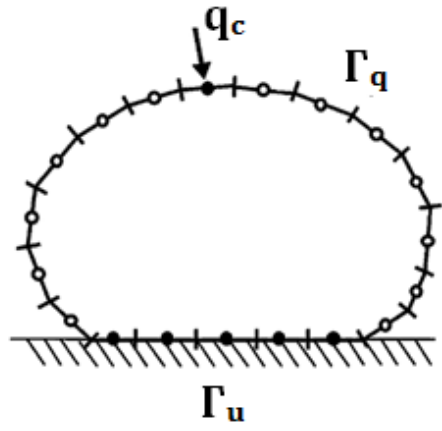

Figura 1: Discretização do contorno de um domínio bidimensional.

Os problemas com domínio infinito ou semi-infinito (domínios abertos) são muito importantes na engenharia. Podem ser citadas aplicações importantes na análise de perfis de escoamento de fluidos em aerofólios, na definição do perfil térmico no entorno de aletas, na identificação do potencial de proteção catódica em tubulações submersas e muitas aplicações importantes na área de geotécnica, como a identificação da integridade estrutural das galerias escavadas para uma mina subterrânea. A Terra, por exemplo, pode ser considerada um meio semi-infinito 
na determinação da variação de temperatura perto de sua superfície. Entre muitos outros exemplos, uma parede espessa pode ser modelada como um meio semi-infinito, se tudo o que interessa é a variação de temperatura na região próxima a uma das superfícies, e a outra superfície é muito distante para influenciar a região de interesse, durante o tempo de observação. Muitas aplicações referentes a domínios infinitos, também podem se relacionar aos casos semi-infinitos, como o problema da proteção catódica de estruturas submersas, por exemplo. Além disso, considerando o exemplo citado, em que a superfície da Terra constitui um caso de meio semi-infinito, existem numerosas e importantes situações onde se deseja fazer uma relação de interface entre um sólido e uma estrutura (e também fluido-estrutura). A teoria de meios semiinfinitos do MEC se aplica com propriedade nestas condições, que incluem a geofísica e a análise sísmica.

Cabe ressaltar, todavia, que não há material abundante sobre a teoria relativa à aplicação do MEC a estes importantes problemas. Nas poucas referências bibliográficas [3-5] não é feito o necessário detalhamento metodológico, excluindo-se o trabalho de Brebbia e Telles [6], que se refere à elasticidade. Isto traz uma importante valorização a este trabalho, pois são muitas as peculiaridades, especialmente no tratamento de problemas com regiões semi-infintas.

\section{A condição de regularidade}

A equação integral do MEC para problemas estacionários abertos pode ser obtida considerando-se um contorno interno $\Gamma_{0}$, envolvido por um círculo de raio $\rho$ centrado em $\xi$, cujo contorno é designado por $\Gamma_{\infty}$ e está infinitamente distante do contorno interno, conforme mostra a Fig. 2. Nesta dedução, tomou-se apenas um contorno interno; porém, a região de interesse pode comportar duas ou mais cavidades, cujo equacionamento segue o mesmo mostrado para esta situação mais simples.

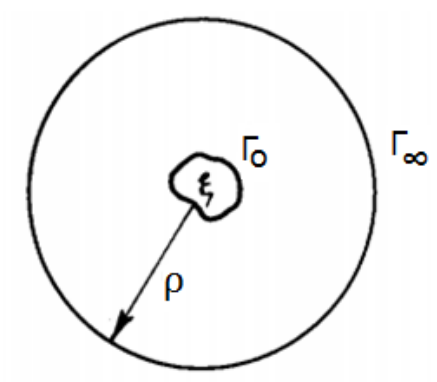

Figura 2: Região delimitada pelo círculo de raio $\rho$ envolvendo uma cavidade.

A equação integral na forma inversa, em princípio, envolve o contorno interno e o contorno infinitamente distante, conforme exposto a seguir:

$$
\begin{gathered}
\mathrm{C}(\xi) \mathrm{u}(\xi)+\int_{\Gamma_{0}} \mathrm{uq}^{*} \mathrm{~d} \Gamma_{0}-\int_{\Gamma_{0}} \mathrm{qu}^{*} \mathrm{~d} \Gamma_{0}= \\
\int_{\Gamma_{\infty}} \mathrm{qu}^{*} \mathrm{~d} \Gamma_{\infty}-\int_{\Gamma_{\infty}} \mathrm{uq}^{*} \mathrm{~d} \Gamma_{\infty}
\end{gathered}
$$

Na Eq. (1), u é a variável primal e q a sua derivada normal; $u^{*}$ é a solução fundamental $e^{*} q^{*}$ sua derivada normal.

Pode-se simplificar a expressão (1) caso os pontos fonte se situem exclusivamente na região de interesse e a seguinte condição for satisfeita:

$$
\lim _{\rho \rightarrow \infty} \int_{\Gamma_{\infty}}\left[q u^{*}-u q^{*}\right] d \Gamma_{\infty}=0
$$

Esta é a chamada Condição de Regularidade, que pode ser obtida nos casos em que se caracterizar o Princípio de Saint-Venant [7], que afirma que u e q tomam o mesmo comportamento da solução fundamental $u^{*}$ e da sua derivada direcional $q^{*}$ em regiões suficientemente distantes do ponto de aplicação da fonte ou carregamento térmico concentrado. Assim, se o carregamento é aplicado numa área de interesse, a certo distanciamento desta região o comportamento do potencial reproduz o comportamento da solução fundamental.

Nestas condições, para os problemas de campo escalar bidimensionais, pode-se verificar que a Eq. (2) é satisfeita, pois as duas parcelas envolvidas se cancelam mutuamente quando $\varsigma \rightarrow \infty$. A condição de regularidade também é obedecida, mas de modo diferente, quando a solicitação aplicada for auto equilibrada. Neste caso, pode-se verificar que o decaimento do potencial é mais acentuado, de modo que as parcelas da Eq. (2) se anulam separadamente. Um único caso de desobediência à condição de regularidade surge quando as condições prescritas em $\Omega$ forem tais que imponham uma uniformidade no potencial em todo domínio. Contudo, este não é um caso prático.

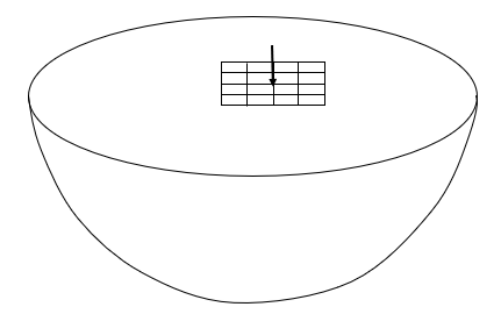

Figura 3: Ilustração de uma região semi-infinita.

Tal como os problemas de domínio infinito, um problema semi-infinito consiste de um domínio idealizado que possui uma superfície plana única e se estende até o infinito em todas as direções, como ilustra a Fig. 3.

\section{O Método das Imagens}


Embora a base teórica seja a mesma, fundamentada na condição de regularidade, no passado o tratamento de problemas semi-infinitos era muito menos eficiente do que nos casos infinitos, pois erros significativos na solução eram introduzidos pela falta de uma solução fundamental adequada. Isto era apenas minimizado pelo uso de elementos de contorno infinitos ou que possuíam interpolação especial. A solução efetiva deste problema foi feita com um ajuste na solução fundamental, feita através do Método das Imagens [4].

O Método das Imagens (MDI) é uma ferramenta matemática usada para resolver equações diferenciais nas quais o domínio da variável básica é estendido pela adição de sua imagem refletida em relação a um plano de simetria. Em decorrência disso, obedece-se automaticamente a certas condições de contorno, facilitando bastante a solução do problema. O MDI é usado em eletrostática para calcular a distribuição do campo elétrico de uma carga nas proximidades de uma superfície condutora e também pode ser usado em magnetostática para calcular o campo magnético de um ímã que está próximo a uma superfície supercondutora [8]. Sua aplicação também ocorre no estudo da reflexão ou absorção de uma pluma contaminante de um contorno dito impenetrável, sem fluxo. Outra aplicação de grande importância está ligada ao estudo e contenção da propagação do som ao ar livre, onde barreiras acústicas são modeladas como corpos sem espessura e neste caso, sua aplicação no contexto do MEC revela grande adequação e eficiência [9].

$\mathrm{Na}$ literatura referente ao MEC, o Método das Imagens foi apresentado inicialmente abordando problemas de potencial [10]. Posteriormente, outras aplicações escalares e em casos da elasticidade foram publicadas $[2,11]$. Há uma relação próxima entre o MDI e as Funções de Green [12], de forma que a utilização do MDI no MEC pode ser interpretada como técnica para adaptação da solução fundamental clássica, de forma que se possam resolver problemas específicos relativos às regiões semi-infinitas. Isto acontece porque a solução fundamental para o meio semi-infinito pode ser encontrada adaptando-se a solução clássica para meios fechados, de forma que esta última satisfaça automaticamente a condição de contorno na interface. Deste modo, pode-se dispensar a discretização do semi-plano usando o MEC.

Para se obter a solução fundamental adaptada a partir da ideia do MDI, é necessário considerar as etapas matemáticas que se seguem, que também arrolam considerações físicas sobre o problema.

Considere inicialmente um espaço bidimensional (vide Fig. 4) onde está aplicada uma fonte situada com coordenadas $(\xi ; a)$ e procura-se entender 0 que acontece em outro ponto situado simetricamente a $\mathrm{x}_{1}$, eixo que é definido pela interface. Este ponto, chamado de fonte imagem, está posicionado exatamente a uma distância $2 a$ da fonte original.

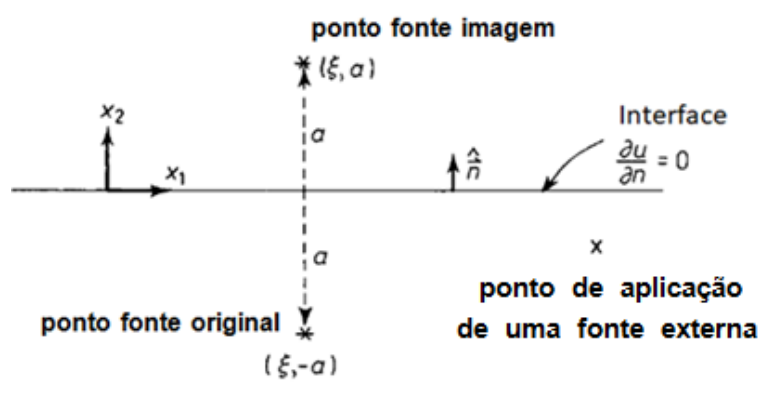

Figura 4: Esquema das fontes simétricas num semi-plano.

Caso se exija a condição de reflexão em $\mathrm{x}_{2}=0$, o campo potencial $\mathrm{mu}^{*}$ produzido pela fonte $\mathrm{m}$ aplicada em ( $\xi$; a) será acompanhado de um efeito similar produzido pela fonte imagem m'a situada na região refletida. Naturalmente, o valor de $\mathrm{m}$ ' depende do tipo de condição de contorno imposto na interface. É do maior interesse a condição de reflexão ou simetria física do potencial, pois esta é muito mais útil às aplicações práticas do MEC. Como a interface está definida ao longo do eixo $\mathrm{x}_{1} \mathrm{e}$ o sentido da normal está no mesmo sentido de $x_{2}$, isto implica que, como condição de contorno de reflexão tem-se:

$$
\frac{\partial \mathrm{u}}{\partial \mathrm{n}}=\frac{\partial \mathrm{u}}{\partial \mathrm{x}_{2}}=0
$$

Assim, resulta a solução fundamental do semi-plano:

$$
\mathrm{u}_{\mathrm{s}}^{*}=\frac{1}{2 \pi} \ln \left[\mathrm{r}_{1}\left(\mathrm{x}_{1} ; \mathrm{a}\right) \mathrm{r}_{2}\left(\mathrm{x}_{1} ;-\mathrm{a}\right)\right]
$$

De posse da solução fundamental, é preciso derivá-la para obter o fluxo fundamental. Percebe-se que esta é uma operação simples, da qual resulta que:

$$
q_{s}^{*}=-\frac{1}{2 \pi} \frac{1}{r_{1}\left(x_{1} ; a\right)}-\frac{1}{2 \pi} \frac{1}{r_{2}\left(x_{1} ;-a\right)}
$$

Ressalta-se que na Eq. (5) os valores de $r_{1}$ e $r_{2}$ tem seu significado bem definidos. São distâncias dos pontos fonte imagem e original aos pontos campo. Em casos em que a interface é retilínea e não haja fontes externas, estas expressões do fluxo fundamental se anulam na interface se os pontos campo são situados ao longo desta, conforme mostra a Fig. 5 .

Percebe-se que, nesta condição, as distâncias $r_{1}$ e $r_{2}$ são iguais; mas, pelos pontos estarem situados simetricamente ao semi-plano, as expressões de $\mathrm{q}^{*}$, embora se somem, acabam se anulando devido ao sinal oposto da normal ser diferente em cada caso. Tal situação acontece na maior parte das aplicações em problemas de eletromagnetismo, área esta em que o MDI é extensamente utilizado. 


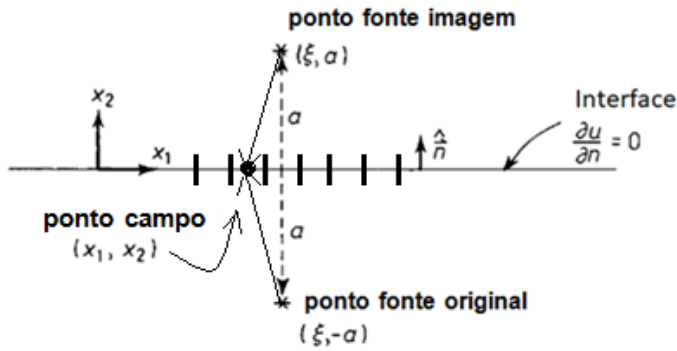

Figura 5: Ponto campo situado na interface e fontes simétricas no interior do semi-plano.

O valor de $\mathrm{q}^{*}$ também se anula no caso em que os pontos fonte e campo estão ambos sobre a interface, conforme mostrado na Fig. 6 a seguir:

ponto fonte imagem $=$ ponto fonte original

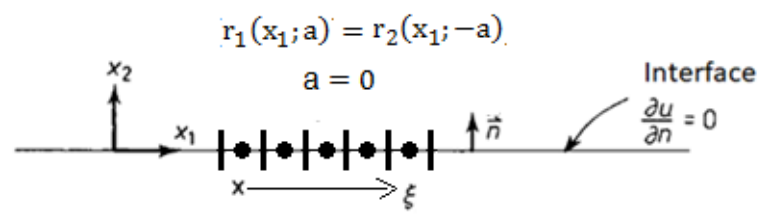

Figura 6: Ponto campo e pontos fonte situados na interface.

Situação bem distinta acontece se há alguma irregularidade no semi-plano, vide Fig. 7, pois neste caso $r_{1}$ é diferente de $r_{2} e$, consequentemente, $q^{*}$ não se anula no contorno curvo:

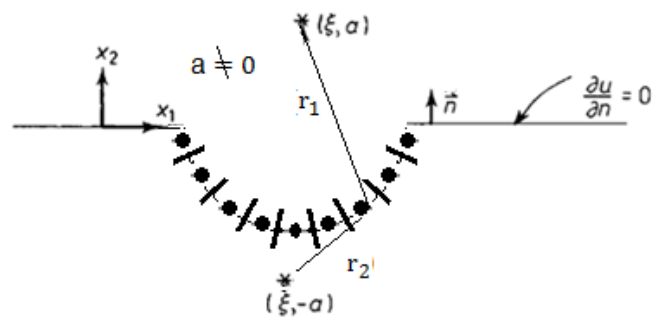

Figura 7: Ponto campo e pontos fonte situados no interior.

O caso mais comum em acústica é quando existe uma fonte sonora, cujos efeitos devem ser atenuados para evitar ressonância e produzir conforto acústico. Nesta situação, vide Fig. 8, é preciso também introduzir uma fonte concentrada, elemento este relativamente simples de se introduzir com o MEC.

\section{Equação integral do MEC para meios semi-infinitos}

A equação integral do MEC estudada para os meios infinitos se mantém no modelo referente aos meios semi-infinitos, incluindo as condições de regularidade, que são obedecidas. Em outras palavras, a solução fundamental do semi-espaço também garante a condição de que tudo o que estiver infinitamente distante pode ser desprezado em razão do decaimento de $\mathrm{u}^{*}$ e $\mathrm{q}^{*}$. Contudo, há aspectos importantes quando se obtém a solução fundamental modificada pelo MDI.

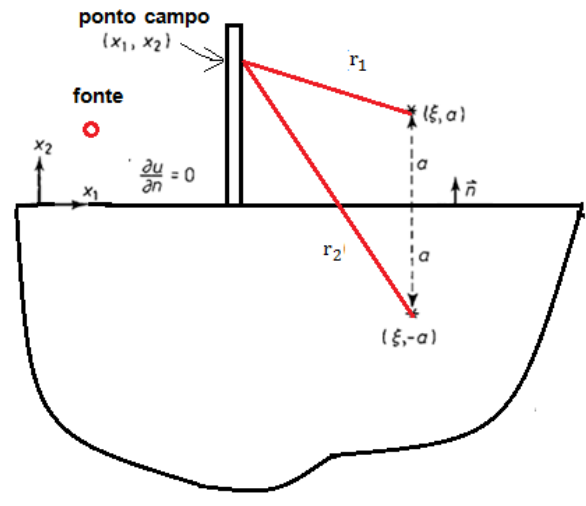

Figura 8: Caso comum envolvendo fonte sonora. Pontos campo e fontes situados fora da interface.

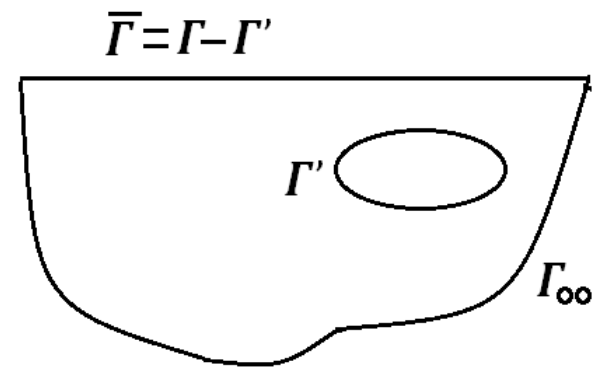

Figura 9: Meio semi-infinito com uma região interna $\Gamma^{\prime}$.

Primeiramente, considere o meio semi-infinito conforme apresentado na Fig. 9. Em muitos casos, a região de interesse não é a superfície definida pelo semi-plano, mas sim uma região interna $\Gamma^{\prime}$, como ocorre nos casos de análise de galerias escavadas numa mina em pouca profundidade. Então, tanto o contorno da interface quanto o contorno interno podem estar carregados. A equação integral do MEC neste caso fica:

$$
c(\xi) u(\xi)+\int_{\Gamma} q^{*} u d \Gamma-\int_{\Gamma} u^{*} q d \Gamma=0
$$

Pois q* é nulo na interface plana, ou seja:

$$
\int_{\bar{\Gamma}} \mathrm{q}^{*} \mathrm{ud} \Gamma=0
$$

Assim, consequentemente, quaisquer condições de Dirichlet não são aplicáveis na interface. O mais importante, contudo, é que apenas as partes do contorno do semi-plano que estiverem sob as condições de fluxo não nulo precisam ser discretizadas, pois a solução fundamental se encarrega de eliminá-las do modelo. A singularidade nos argumentos da solução fundamental e sua derivada normal no caso de coincidência entre os pontos fonte e campo também são resolvidos do mesmo modo que no MEC clássico 
para meios fechados, pois $\mathrm{u}^{*}$ é uma singularidade logarítmica, que pode ser integrada no sentido usual. Seu integrando é impróprio, mas como se trata de uma singularidade fraca, este é integrável. A singularidade em $\mathrm{q}^{*}$ não existe, pois, a própria solução fundamental foi gerada com base na anulação da sua derivada na interface. Assim, o procedimento padrão do MEC se aplica a estes problemas, ou seja, após a discretização é gerada uma forma matricial clássica para o caso dos pontos campo estarem situados numa parte curva da interface, ou seja:

$$
[\mathrm{H}](\mathrm{U})=[\mathrm{G}](\mathrm{Q})
$$

Na eq. 8, U e $Q$ são matrizes linha de potencial e fluxo, contendo valores nodais conhecidos e também valores a serem calculados. $\mathrm{G}$ e $\mathrm{H}$ são matrizes provenientes das integrais de ponderação para o potencial e fluxo, respectivamente. O mesmo acontece se existem pontos campo e cargas num contorno interno qualquer, próximo da interface. Neste caso, também a quantidade de pontos fonte deve ser igual à quantidade de pontos campo.

No caso de aplicações com contornos retos e cargas apenas neste aplicadas, visando a determinação de potenciais ou fluxos fora da interface, as integrais contendo $\mathrm{q}^{\star}$ se anulam - a matriz $\mathrm{H}$ desaparece - e o sistema fica apenas na forma:

$$
[\mathrm{I}](\mathrm{U}(\xi))=[\mathrm{G}](\mathrm{Q})
$$

$\mathrm{Na}$ Eq. (9), I é a matriz identidade. Neste caso o coeficiente $c(\xi)$ é determinado automaticamente pela forma da solução fundamental, que aparentemente se degeneraria no dobro da solução fundamental clássica. Porém, isso não ocorre: um ponto fonte está no interior $(c(\xi)=1)$ e o outro fora da região $(c(\xi)=0)$.

Ressalta-se que a obtenção de valores do potencial na interface acontece sem a necessidade de resolver qualquer sistema de equações, o que é uma enorme vantagem computacional. Obviamente, o mesmo vale para o cálculo dos valores do potencial ou fluxo, caso se queira, em pontos situados no interior do domínio semi-infinito.

\section{Simulações numéricas}

\subsection{Carga concentrada numa superfície reta}

Neste primeiro exemplo aplica-se um fluxo constante num trecho bastante reduzido de uma interface retilínea, conforme mostra a Fig. 10. Ressalta-se que os elementos de contorno são constantes e dessa forma o carregamento aplicado não se estende aos elementos vizinhos, cuja condição prescrita é fluxo nulo. O interesse aqui é reproduzir o problema fundamental. É possível reduzir ainda mais o intervalo, considerando apenas um elemento, mas do modo apresentado já será possível perceber que a solução deste caso em pontos internos se aproxima do comportamento dado pela solução fundamental, ajustada para um valor do fluxo que não é unitário, é igual a 20 .

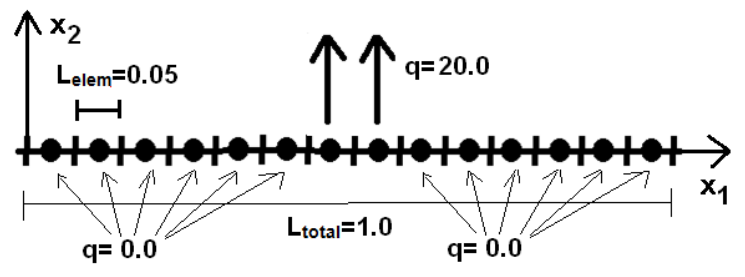

Figura 10: Carga concentrada aplicada numa superfície reta.

Tabela 1: Temperatura no contorno - Exemplo 1.

\begin{tabular}{ccc}
\hline \multicolumn{1}{c}{ Tabela 1: Temperatura no contorno - Exemplo 1. } \\
\hline 0.025000 & $\mathbf{x}_{\mathbf{2}}$ & Potencial \\
\hline 0.075000 & 0.000000 & -0.475105 \\
\hline 0.125000 & 0.000000 & -0.546209 \\
\hline 0.175000 & 0.000000 & -0.626312 \\
\hline 0.225000 & 0.000000 & -0.718046 \\
\hline 0.275000 & 0.000000 & -0.954936 \\
\hline 0.325000 & 0.000000 & -1.118491 \\
\hline 0.375000 & 0.000000 & -1.341674 \\
\hline 0.425000 & 0.000000 & -1.704284 \\
\hline 0.475000 & 0.000000 & -2.443571 \\
\hline
\end{tabular}

Os resultados de temperatura no contorno são apresentados na Tabela 1. Se o fluxo fosse efetivamente singular, o valor do potencial seria infinito. A tática aqui empregada para avaliar a precisão destes resultados no contorno é calcular os valores do potencial em pontos internos - cuja metodologia se baseia no Método das Imagens - em pontos ligeiramente afastados do contorno, mas que estão equidistantes destes. Para melhor ilustrar 0 procedimento exposto, a Fig. 11 mostra um potencial uniformemente distribuído num semicírculo.

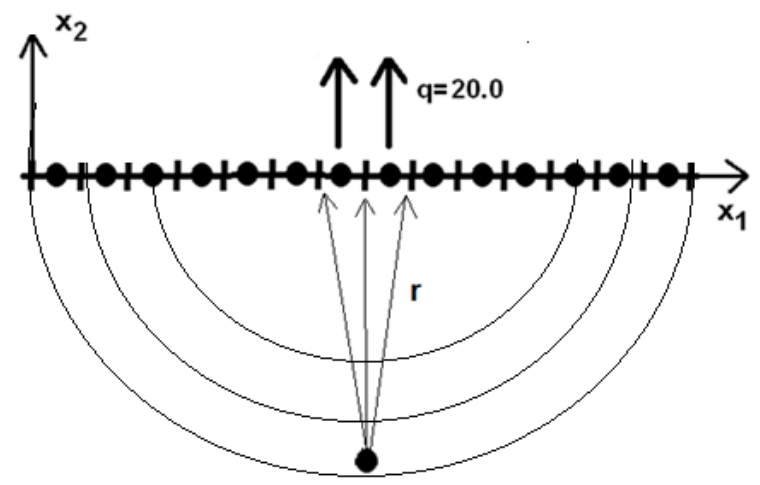

Figura 11: Potencial uniformemente distribuído num semicírculo. 
A Tabela 2 apresenta os resultados desta comparação entre valores calculados internamente e no contorno. Pode-se perceber que há uma concordância bastante satisfatória nos resultados. Um maior erro foi observado apenas no valor do potencial referente ao último nó, a partir do qual a discretização foi truncada. Deve-se considerar ainda que os valores obtidos internamente se calculam através do reuso da equação integral, o que resulta em valores numéricos de maior precisão comparativamente aos valores calculados nos nós de contorno [13,14].

\begin{tabular}{cccc}
\multicolumn{4}{l}{ Tabela 2: Pontos equidistantes dos nós de contorno. } \\
\hline $\mathbf{x}_{1}$ & $\mathbf{x}_{2}$ & $\begin{array}{c}\text { Potencial } \\
\text { interno }\end{array}$ & $\begin{array}{c}\text { Valor no } \\
\text { contorno }\end{array}$ \\
\hline 0.500 & -0.175 & -1.101152 & -1.118491 \\
\hline 0.500 & -0.375 & -0.622539 & -0.626312 \\
\hline 0.500 & -0.500 & -0.440214 & -0.475105 \\
\hline
\end{tabular}

$\mathrm{Na}$ Tabela 3 é feita a comparação entre os valores analíticos, baseados no comportamento da solução fundamental e valores em pontos internos situados ao longo da reta $x_{1}=0.5$. Nestes pontos, os valores ditos analíticos foram calculados considerando que a partir de uma distância razoável da superfície livre, o raio vetor $r$ varia pouco, uma vez que o setor carregado é bastante restrito e erros de integração são pouco importantes. Observa-se que, apesar das restrições mencionadas para tal comparação, há uma concordância muito boa entre os resultados analíticos e numéricos.

Tabela 3: Potencial nos pontos internos.

\begin{tabular}{cccc}
$\mathbf{x}_{\mathbf{1}}$ & $\mathbf{x}_{\mathbf{2}}$ & $\begin{array}{c}\text { Potencial } \\
\text { interno }\end{array}$ & $\begin{array}{c}\text { Potencial } \\
\text { analítico }\end{array}$ \\
\hline 0.50 & -0.50 & -0.440214 & -0.441270 \\
\hline 0.50 & -1.00 & 0.000265 & 0.000000 \\
\hline 0.50 & -2.00 & 0.441338 & 0.441270 \\
\hline 0.50 & -5.00 & 1.024611 & 1.024600 \\
\hline
\end{tabular}

\subsection{Potencial uniformemente distribuído num semicírculo}

Numericamente, este exemplo se distingue bastante do anterior, pois condições de contorno envolvendo tanto potencial quanto ou fluxo podem ser aplicadas, porque são prescritas num contorno que não pertence à interface. A Fig. 12 ilustra o problema proposto: trata-se do caso em que um potencial uniforme e unitário de temperatura é aplicado ao longo do semicírculo de raio igual a dois.

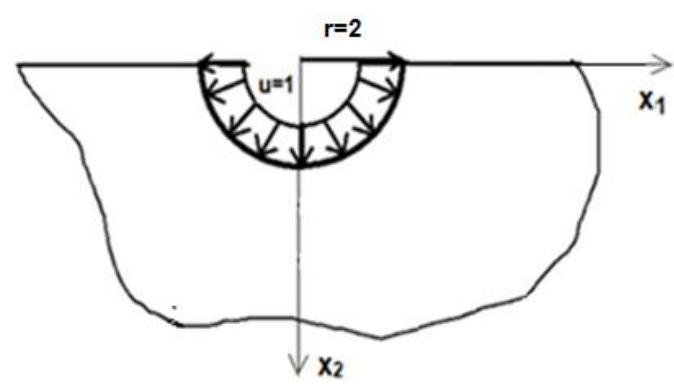

Figura 12: Potencial uniformemente distribuído num semicírculo.

Quando não existem fluxos aplicados na interface e a região de interesse lhe é simétrica, este problema é equivalente a parte inferior (ou superior) de um problema com domínio infinito. No caso, existe uma solução simples para comparação, que é baseada no comportamento da solução fundamental.

Conforme mencionado, a discretização fica limitada ao contorno semicircular. Neste caso, diferentemente dos casos anteriores, ambas as matrizes $\mathrm{H}$ e $\mathrm{G}$ são geradas. A Fig. 13 ilustra o modelo discreto adotado, em que pontos fonte imagem são posicionados simetricamente aos pontos fonte usuais do MEC.

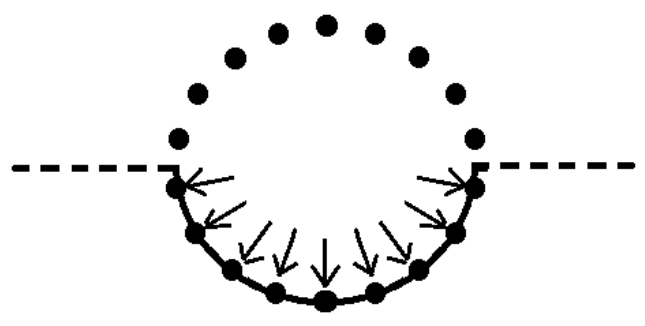

Figura 13: Discretização do orifício em meio semi-infinito.

O sistema matricial a ser resolvido é:

$$
[\mathrm{G}](\mathrm{Q})=[\mathrm{H}](\overline{\mathrm{U}})=(\mathrm{B})
$$

Assim, apenas os fluxos são calculados no contorno. Ressalta-se que a solução fundamental e sua derivada normal, referentes ao semi-plano devem ser utilizadas neste caso e em problemas similares, pois tais funções auxiliares permitem que não se necessite fazer qualquer discretização ao longo da interface, pois a condição de simetria é naturalmente incorporada ao problema.

A solução analítica do problema em meio infinito com raio $r=2$ e o uso da solução fundamental clássica aponta um valor de fluxo no contorno igual a 0.72135 . O modelo discreto aqui utilizado, com 20 elementos de contorno constantes, resultou num valor igual a 0.713453, com erro relativo percentual aproximadamente de 1\%. Embora, bem próximos, tais valores numéricos certamente podem ser reduzidos com o refinamento da malha, eliminando assim erros 
na representação da geometria circular e problemas nos cantos.

Para os pontos internos, os valores numéricos obtidos em pontos afastados também estão satisfatórios e estão mostrados na Tabela 4 a seguir, acompanhados dos valores analíticos. Em termos numéricos, deve-se ressaltar que devido aos pontos fonte originais estarem sobre o contorno, os coeficientes da diagonal da matriz $\mathrm{H}$ referentes à integração com base nestes pontos é igual a 0.5 , pois os elementos são constantes. Já os coeficientes gerados pelos pontos fonte de imagem têm $c(\xi)$ iguais à zero, pois tais pontos estão fisicamente no vazio.

Nos casos em que o problema apresenta uma simetria vertical, pode-se constatar que os coeficientes $\mathrm{H}$ são praticamente nulos, quando da obtenção dos valores em pontos situados sobre este eixo vertical de simetria.

Tabela 4: Valores numéricos obtidos em pontos afastados do contorno.

\begin{tabular}{cccc}
\hline $\mathbf{x}_{1}$ & $\mathbf{x}_{\mathbf{2}}$ & $\begin{array}{c}\text { Potencial } \\
\text { numérico }\end{array}$ & $\begin{array}{c}\text { Potencial } \\
\text { analítico }\end{array}$ \\
\hline 0.00 & -3.00 & 1.567359 & 1.583460 \\
\hline 0.00 & -4.00 & 1.977610 & 1.997700 \\
\hline 0.00 & -5.00 & 2.295847 & 2.319110 \\
\hline 0.00 & -6.00 & 2.555877 & 2.582550 \\
\hline
\end{tabular}

\section{Conclusões}

O presente trabalho apresentou com clareza as características teóricas e numéricas referentes à abordagem do MEC aos problemas com domínio aberto, destacando particularmente as condições de regularidade no infinito, que permitem que sejam desprezados na equação integral de governo os efeitos de pontos infinitamente distantes da região de interesse. Destacou também o papel da solução fundamental obtida pelo Método das Imagens, importante para que a discretização de problemas semi-infinitos seja muito simples e se possam desprezar condições de Dirichlet na interface.

Pode-se destacar a simplicidade com que o MEC formula e resolve os problemas citados, demandando limitada quantidade de pontos nodais para a devida discretização.

Dois problemas típicos foram resolvidos, nos quais a tática de abordagem foi apresentada claramente e os resultados numéricos obtidos mostraram precisão satisfatória, ratificando que o MEC é a técnica mais adequada ainda hoje para resolver tais problemas.

\section{Referências}

[1] BREBBIA, C. A.; DOMíNGUEZ, J. The Boundary Element Method - An Introductory. Course. WIT Press, 1998.

[2] BREBBiA, C. A.; TELLES, J. C. F.; WROBEL, L. C. Boundary Element Techniques. SpringerVerlag, Berlin, 1984.

[3] DAVIES, T. G.; BU, S. Infinite Boundary Elements for the Analysis of Halfspace Problems. Computer and Geotechnics, v. 19, n. 2, p. 137- 151, 1996.

[4] GAO, X. W.; DAVIES, T. G. 3-D Infinite Boundary Element for half-space problems. Engineering Analysis with Boundary Elements, v. 21, p. 207-213, 1998.

[5] SIMÕES, N.; TADEU, A. Fundamental solutions for transient heat transfer by conduction and convection in an unbounded, half-space, slab and layered media in the frequency domain. Engineering Analysis with Boundary Elements, v. 29, p.1130-1142, 2005.

[6] TELLES, J. C. F.; BREBBIA, C. A. Boundary element solution for half-plane problems. Int. J. Solids Structures, v. 17, n. 12, p. 1149-1158, 1981.

[7] TIMOSHENKO, S. Teoria da Elasticidade, Guanabara Dois, Rio de Janeiro, 1980.

[8] OLIVEIRA M. T.; PAGAN, C. J. B. The Method of Images applied to the grounded sphere: The problem of the ground wire. Journal of Electrostatics, v.70, p. 292299, 2012.

[9] SOENARKO, B.; SETIADIKARUNIA, D. An analytical solution versus half space BEM formulation for acoustic radiation and scattering from rigid sphere. Bandung: Journal of Physics, 2016, Conference Series, 7760120652016.

[10] BREBBIA, C. A.; WALKER, S. Boundary Element Techniques in Engineering. Newnes-Butterworths, London, 1980.

[11] PAN, E.; CHEN, C.; AMADEI, B. A BEM formulation for anisotropic half-plane problems. Engineering Analysis with Boundary Elements, v. 20, p.185-195, 1997.

[12] BAETA SEGUNDO, J. A. O Método das Imagens e as Funções de Green. Revista Brasileira de Ensino de Física, v. 20, n. 2, 1998.

[13] FREITAS, A. B.; LOEFFLER, C. F. Performance evaluation of the boundary element recursive procedure in elastic problems. J Eng Math, v. 98, p. 11-20, 2016.

[14] RAMOS, V. E. S.; LOEFFLER, C.F.; MANSUR, W.J. Recursive Procedure of Boundary Element Method Applied to Poisson's Problems. Eng. Analysis with Boundary Elements, v. 82, p. 104-110, 2017. 